\title{
Beyond similarity: Masking of the target is sufficient to cause the attentional blink
}

\author{
TIMOTHY D. GRANDISON, THOMAS G. GHIRARDELLI, and HOWARD E. EGETH \\ Johns Hopkins University, Baltimore, Maryland
}

\begin{abstract}
When subjects are asked to identify a letter target embedded in a rapid serial visual presentation stream, the detection of a subsequent letter probe is briefly impaired. This transient deficit in probe detection, termed the "attentional blink," depends on the type of item that immediately follows the letter target (Raymond, Shapiro, \& Arnell, 1995). Two models have been proposed to account for this effect. The interference model of the attentional blink predicts that visual similarity between the probe and item immediately following the target $(+1 \mathrm{item})$ causes the attentional blink, whereas the two-stage model is based on the notion that increased time needed to process the target letter causes the attentional blink. In order to test between these two possibilities, the masking properties of the +1 item and its similarity to the probe were varied. We found the attentional blink when the +1 item acted as a mask of the target, even though the +1 item and the probe were visually dissimilar. This pattern of results supports the two-stage model of the attentional blink.
\end{abstract}

Much of the past work on visual attention has focused on how visual attention is deployed across space. Recently, however, a number of studies using rapid serial visual presentation (RSVP) have explored how attention is deployed over time. In the RSVP paradigm, a series of items (individual letters, words, or pictures) is presented sequentially at a rate of 6 to 20 items $/ \mathrm{sec}$. Subjects are typically required to identify one or more target items that are distinguished from the rest of the RSVP stream on the basis of either feature information (Broadbent \& Broadbent, 1987; Raymond, Shapiro, \& Arnell, 1992; Shapiro, Raymond, \& Arnell, 1994) or category information (Broadbent \& Broadbent, 1987; Chun \& Potter, 1995).

When the RSVP stream contains two targets, identification of the second target is sometimes impaired. This was first shown by Broadbent and Broadbent (1987), who had subjects identify uppercase words that were embedded in a stream of lowercase words. Accuracy in identifying the first target was high, but identification of the second target was impaired when the two targets appeared within $400 \mathrm{msec}$ of each other. Similar results are obtained when the targets are letters instead of words (Raymond et al., 1992; Weichselgartner \& Sperling, 1987). In Raymond et al.'s Experiment 2, subjects were instructed to complete two tasks. The first was the identification of a white letter (the target) presented in a stream of black letters. The second task involved reporting whether or not a black $X$ (the probe) was present on a given trial. The probe was presented on $50 \%$ of the trials and could occur

This research was supported by NSF Grant SBR-9319356 awarded to H.E.E. We are grateful to Vincent DiLollo, Chip Folk, Art Kramer, and Jane Raymond for their helpful comments. Correspondence should be addressed to T. Grandison, Department of Psychology, Johns Hopkins University, Baltimore, MD 21218 (e-mail: tim@ren. psy.jhu.edu). in any one of eight posttarget positions. Note that here the word position refers to a location in time rather than one in space. Subjects were significantly impaired in detecting the probe when it appeared from 180 to $450 \mathrm{msec}$ after the target. No such deficit was observed when the probe immediately followed the target, or when more than $450 \mathrm{msec}$ had elapsed between the presentation of the target and the appearance of the probe. This transient deficit in probe detection was termed the "attentional blink" (Raymond et al., 1992). Explanations of a sensory nature (i.e., masking) were ruled out through the use of a control condition that used the exact same displays as described above but with an instruction to subjects to ignore the white letter and just perform the probe detection task. In this condition, subjects accurately detected the probe regardless of its position relative to the target. Thus, probe detection was impaired only when subjects had attended to and processed a previous target item.

Several findings suggest that the item immediately following the target (called the "+1 item") plays an important role in determining whether or not the attentional blink occurs (Chun \& Potter, 1995; Raymond et al., 1992). In one experiment, for example, Raymond et al. used the procedure described above with one exception. Instead of presenting a letter in the +1 position, they replaced the +1 item with a blank interval. During this blank interval, the entire screen was filled only with the background color. This manipulation did away with the attentional blink. That is, the accuracy level of probe detection was uniformly high and did not depend on the posttarget position of the probe, suggesting that a +1 item is necessary to produce the attentional blink (Raymond et al., 1992). This finding was subsequently replicated by Chun and Potter.

The magnitude of the attentional blink is indexed by the size of the reduction in the accuracy of identifying the 
probe. Studies have shown that the amount of similarity between the target and the +1 distractor influences the size of the attentional blink. For example, Chun and Potter (1995) required subjects to identify two letters embedded in a stream of items. For a given stream, the nontarget items could be digits, symbols, or a combination of digits and symbols. The first letter (the target) could be immediately followed by either a symbol or a digit. The digits in these experiments were more similar to the letter targets than were the symbols. When the target was followed by a digit, the accuracy of identifying the second letter (the probe) was reduced in the pattern typical of the attentional blink. When the target was followed by a symbol, the pattern of results was similar, but probe identification was significantly better.

Raymond et al. (1995) conducted a series of experiments that also varied the similarity of the target and the $+1 \mathrm{item}$. As in previous experiments, the subjects viewed an RSVP stream of letters and then had to identify the target and detect the presence of the probe. The +1 item could be a digit, the letter $\mathrm{S}$, a dot pattern, or a displaced letter that was presented $.82^{\circ}$ to the right of the target. When the featural or spatial similarity between the target and the +1 item was decreased, the attentional blink was also reduced. Changing the +1 item from a letter to a digit had no significant effect on the magnitude of the attentional blink, which led the authors to conclude that categorical similarity does not affect the attentional blink. Note that because Raymond et al. (1995) and Chun and Potter (1995) used letters as both the target and the probe, varying the similarity between the target and the +1 item also had the effect of varying the similarity between the +1 item and the probe. This point is important when one is evaluating the models discussed below.

A number of models have been put forth to explain the attentional blink. Raymond et al. (1995) described the interference model, which is an adaptation of similarity theory (Duncan \& Humphreys, 1989), and proposes that the attentional blink arises after perceptual descriptions of the RSVP items have entered into a visual short term memory (VSTM) buffer. Depending on how well items match target or probe templates, they are assigned weightings in VSTM. These weightings are a limited resource and degrade over time. According to this model, the attentional blink results from the following sequence of events. Initially, a perceptual description of the target gains entry into VSTM and is assigned a high weighting because of its close match to the target template. The +1 item, because of its temporal proximity to the target, also enters VSTM. In addition, the perceptual description of the +1 item receives a higher weighting if it is similar to the probe template. When the probe appears, it also gains entry to VSTM because of its match with the probe template. If the probe appears soon after the +1 item, its weighting is limited by the amount of weighting the target and the +1 item have already received. When the probe does not receive a high enough weighting, errors in its retrieval will occur, thus causing the attentional blink. As the delay be- tween the target and the probe increases, the weightings given to the target and the +1 item decay. This allows the probe to receive a higher weighting, and thus it can be retrieved accurately from VSTM. Because the +1 item receives a high weighting to the extent that it matches the probe template, similarity between the +1 item and the probe causes the attentional blink.

In contrast to the above model, various multiple-stage models have been used to explain the attentional blink that do not rely on limitations based on retrieval from memory (Broadbent \& Broadbent, 1987; Chun \& Potter, 1995; Raymond et al., 1992; Weichselgartner \& Sperling, 1987). Out of these models, the one proposed by Chun and Potter is both well specified and best fits the existing data, and for these reasons it will be considered in more detail. In the first stage of this model, items are detected and short-lived representations of them are formed that allow for the selection of candidate targets for further processing. Unless selected for further processing, subsequent items overwrite this initial representation. These initial representations must undergo further processing in order for identification to occur. This additional second-stage processing is limited in capacity and its duration exceeds the stimulus onset asynchrony. The attentional blink occurs when the second target in an RSVP stream appears before the second-stage processing of the first target is complete. The initial representation of the second target will still be formed, but second-stage processing will be delayed. The longer this delay, the greater the likelihood that the initial representation of the second target will have faded before it can undergo second-stage processing. Chun and Potter assumed that when the +1 item is visually similar to the target, delays occur during second-stage processing, resulting in an increased attentional blink.

Alternatively, this two-stage model can explain the effects of similarity between the target and the +1 items in terms of visual masking. "Visual masking refers to the reduction of visibility of one stimulus, called the target, by a spatiotemporally overlapping or contiguous second stimulus, called the mask" (Breitmeyer, 1984, p. 2). For the purposes of this explanation, the +1 item acts as a spatially overlapping mask for the preceding item. When Raymond et al. (1992) used the previously discussed control condition, they seemed to rule out a masking account of the attentional blink. How, then, is it possible that the masking properties of the +1 item determine the accuracy of probe detection? In the Raymond et al. (1992) experiment, when subjects ignored the first target, they were able to detect the probe accurately, showing that the target, and for that matter, the +1 item, do not mask the probe. This apparent contradiction can be explained in the following way. When the +1 item is presented, it acts as a mask for the target. This increases the amount of time taken to process the target. Just as described above, an increase in the time taken to process the target results in an increase in the likelihood that the probe will be missed. This can be used to explain some of the previous results in the following way. Items that have similar forms tend 
to have similar spatial frequencies. Masks are most effective when they share a similar spatial frequency with the target (White \& Lorber, 1976); therefore, the effects of visual similarity between the target and the +1 item on the magnitude of the attentional blink might be mediated by masking. According to this interpretation of the twostage model, the reason categorical similarity did not influence the attentional blink in Raymond et al. (1995) is that masking is thought to occur at a low level in the visual system; hence categorical similarity would not be predicted to influence masking.

In the experiments, we investigated the effects of immediate posttarget interference resulting from various types of visual stimuli presented as the +1 item. For a preliminary report of these results, see Ghirardelli, Grandison, and Egeth (1995). Similar ideas have been developed independently by Seiffert and DiLollo (in press).

\section{EXPERIMENT 1}

In a pilot study, we found, as Raymond et al. (1995) did, that a +1 item visually similar to the target and yet not a letter could cause the attentional blink. These results, however, can be explained equally well by both an interference model or a two-stage model. In the interference model, it is assumed that the visually similar +1 item occupies resources in VSTM that would otherwise be dedicated to the probe. According to the two-stage model, the +1 item could have acted as a pattern mask on the target. This, then, would have increased the amount of time needed for second-stage processing of the target. It is possible to distinguish between these two possibilities by using $a+1$ item that has masking properties but that is not visually similar to the other letters presented. In the first experiment, a solid white rectangle was used as the +1 item. The white rectangle had an increased luminance relative to the background and hence could act as a luminance mask.

\section{Method}

Subjects. Twenty-five undergraduate students participated in order to partially fulfill a research requirement for an introductory psychology course. All subjects reported normal or corrected-tonormal visual acuity.

Apparatus. The stimuli were generated and displayed on a 13-in. color VGA monitor. Viewing distance was not fixed, but most subjects viewed the display from a distance of approximately $35 \mathrm{~cm}$. Responses were entered on the computer keyboard by the subject.

Procedure. Each subject participated in a single experimental session. Each session consisted of three blocks of 140 trials each. The first block was always practice and the computer gave feedback to the subjects via tones during this block only. Each of the remaining blocks began with 10 practice trials with no feedback. Data were not collected for any of the practice trials. For the remaining blocks, half of the subjects received the experimental block followed by the control block, and the other half of the subjects received the reverse order. Subjects were allowed to rest after the practice block and were required to take a break between conditions.

Each trial consisted of the presentation of a central fixation point followed by a series of successively presented uppercase letters and a pattern mask. The stimuli were presented at a single loca- tion on a light gray background at a rate of $10 \mathrm{items} / \mathrm{sec}$ (33 $\mathrm{msec}$ stimulus duration, $67 \mathrm{msec}$ interstimulus interval [ISI]). The black items had a luminance of $.2 \mathrm{~cd} / \mathrm{m}^{2}$, and the background had a luminance of $69.8 \mathrm{~cd} / \mathrm{m}^{2}$. At a viewing distance of $35 \mathrm{~cm}$, these letters subtended $1.6^{\circ}$ of visual angle vertically and $1.1^{\circ}$ horizontally. Each letter to be presented was randomly chosen from 25 letters (all but $\mathrm{X}$ ) with the additional constraint that no letter be presented twice within a given trial.

On each trial, a letter in a randomly selected temporal position in the letter stream was designated as the target letter, with the constraint that the target letter could not be one of the first 7 letters. The target letter was presented in green (luminance $=57.2 \mathrm{~cd} / \mathrm{m}^{2}$ ), and the remaining items during the trial, except for the white rectangle, were presented in black. The target was preceded by 7 to 15 letters and was always followed by the white rectangle and 7 additional letters. The item immediately following the target was always the white rectangle. The solid white rectangle (luminance $=$ $115.9 \mathrm{~cd} / \mathrm{m}^{2}$ ) subtended $2.5^{\circ}$ of visual angle, both horizontally and vertically. On half of the trials, 1 of the 7 letters that followed the target was an $\mathrm{X}$ (the probe). On the other half of the trials, no $\mathrm{X}$ was presented. In each block, the probe was presented 10 times at each possible temporal position, yielding 70 probe-present trials in each block.

Subjects initiated a trial by pressing a key on the computer keyboard. After the presentation of the RSVP stream, the subject was prompted to respond. The displays in the control and experimental blocks were identical. The only difference between the two blocks was the type of task the subject was required to perform. In the experimental block, subjects were required to identify the target and then detect the probe, whereas in the control condition, the subject merely had to detect the probe. In the experimental blocks, the command "Enter the target letter" appeared on the screen. The subject then typed in the identity of the target letter, after which appeared the question, "Was the probe (black X) present? (Y or N)." In the control condition, only a single question appeared, asking whether or not the probe was present.

\section{Results and Discussion}

In Figure 1, the percentage of trials in which the probe was correctly detected is plotted as a function of the probe's position relative to the target for both the experimental and the control conditions. (For the experimental conditions, probe detection accuracies are based only on the trials in which the target was correctly identified.) A repeated measures analysis of variance (ANOVA; task condition $\times$ probe position) yielded a significant main effect of task condition $[F(1,24)=77.602, p<.001]$, a significant main effect of probe position $[F(6,144)=21.182$, $p<.001]$, and a significant task condition $\times$ probe position interaction $[F(6,144)=15.937, p<.001]$. Subjects correctly detected the probe $91.3 \%$ of the time in the control condition and $73.3 \%$ of the time in the experimental condition. Accuracy for identifying the first target (experimental condition only) was $88 \%$. The presence of a significant main effect of task condition, a significant main effect of probe position, and a significant interaction between task condition and probe position indicates the presence of the attentional blink. Inspection of Figure 1 confirms that the typical attentional blink effect was found. Probe detection performance was better in the control than in the experimental condition. Also, probe position had a larger effect on probe detection accuracy in the experimental condition than in the control condition. 


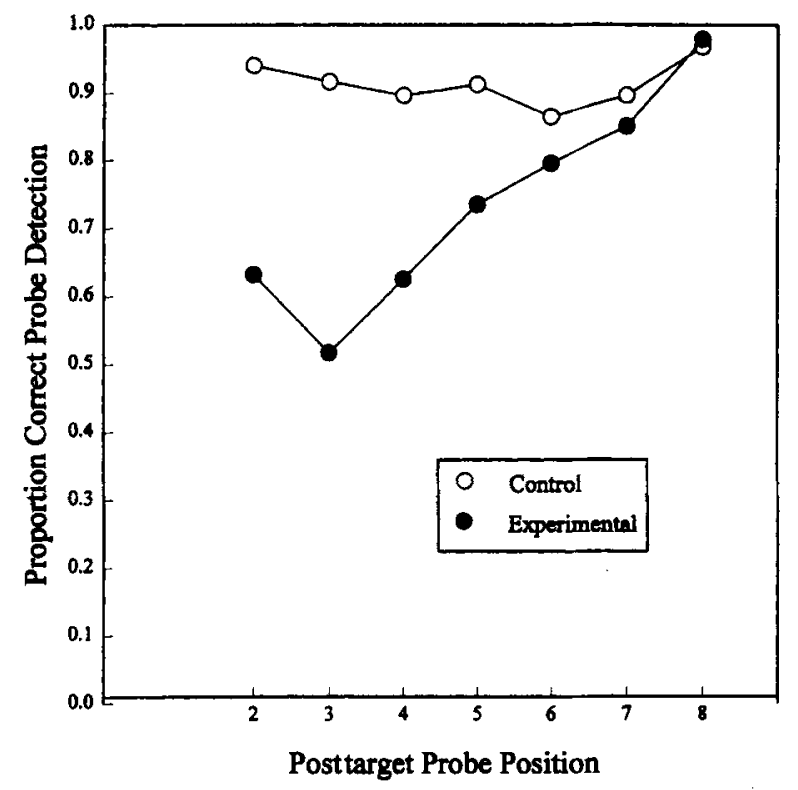

Figure 1. Mean proportion of correct probe detection trials as a function of probe position for both the experimental and the control conditions in Experiment 1. In the experimental condition, correct identification of the target resulted in a marked decrease in probe detection performance, compared to the control condition, when the probe appeared within $\mathbf{5 0 0}$ msec of the target (posttarget Probe Positions 2-5). This is the typical attentional blink effect.

The results of this experiment show that the +1 item can cause the attentional blink even when it is visually dissimilar to both the target and probe.

\section{EXPERIMENT 2}

Experiment 1 demonstrated that the attentional blink can occur even when the +1 item is a white rectangle. The white rectangle is visually dissimilar to the target and probe. We interpreted this result as indicating that a luminance mask can cause the attentional blink. However, it is possible that the white rectangle, which had clearly defined contours, caused the attentional blink because it contained some pattern information. The interference model holds that any patterned stimulus presented subsequent to the target will cause the attentional blink. This is based on the following pattern of results: The attentional blink was found when patterned +1 stimuli, such as a dot pattern, were used (Raymond et al., 1995), but not when a blank was presented in the +1 position (Raymond et al., 1992). According to the interference model, using a blank as the +1 item eliminates the blink because there is no superfluous patterned stimulus stored in VSTM to interfere with subsequent retrieval of the target and probe from VSTM. The two-stage model of the attentional blink explains this by postulating that the blank has no masking properties. It is possible to present a +1 item that has the same amount of pattern information as the blank, but that also has masking properties. In Experi- ment 2 , the +1 item was a white screen flash. It differed from a blank only in its luminance.

\section{Method}

Subjects. Subjects were 17 undergraduate students at Johns Hopkins University who participated in order to partially fulfill a course requirement. None of the subjects in Experiment 2 had participated in the previous experiment. The apparatus and stimuli were identical to those of Experiment 1 with the following exceptions. In Experiment 2, a white screen flash was presented in the +1 position, rather than a white rectangle. The white screen flash had the same luminance as the white rectangle, but differed in spatial extent. The white rectangle of Experiment 1 covered roughly the same area as a letter, whereas the white screen flash covered the entire face of the monitor (approximately $28.5^{\circ}$ of visual angle vertically and $34.7^{\circ}$ horizontally). The target was still green and the items remained black.

\section{Results and Discussion}

Figure 2 is a plot of correct probe detection as a function of probe position for both the control and experimental conditions. A repeated measures ANOVA (task condition $\times$ probe position) revealed a significant main effect of task condition $[F(1,16)=20.809, p<.001]$, a significant main effect of probe position $[F(6,96)=6.713$, $p<.001]$, and a significant task condition $\times$ probe position interaction $[F(6,96)=5.892, p<.001]$. Subjects correctly detected the probe $88 \%$ of the time in the control condition and $78.6 \%$ of the time in the experimental condition. Accuracy for identifying the first target was $94 \%$. Figure 2 demonstrates that, just as in the previous experiment, probe detection performance was better in the control condition than in the experimental condition. Probe position affected probe detection performance more in the experimental condition than in the control condition.

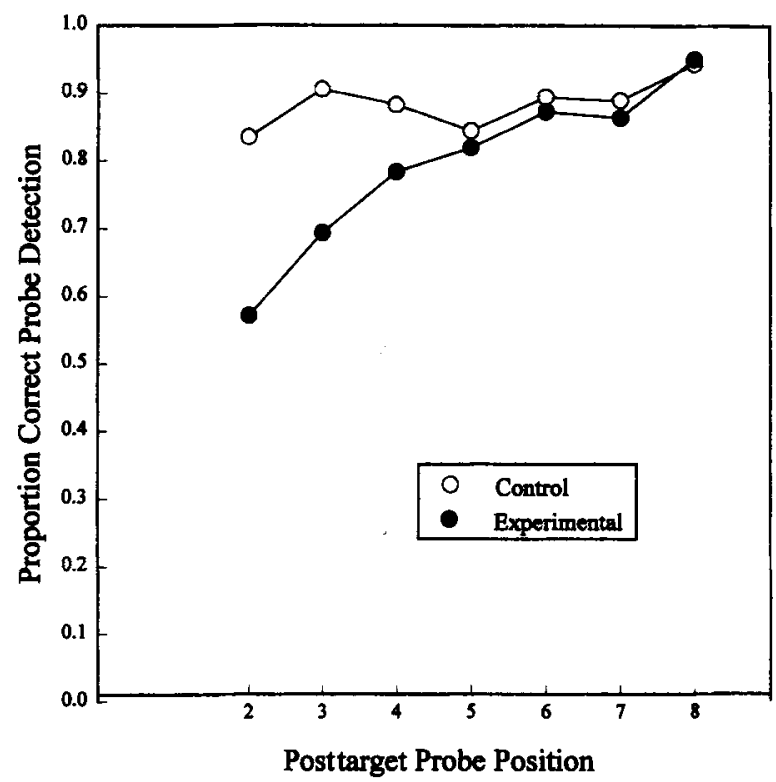

Figure 2. Mean proportion of correct probe detection trials as a function of probe position for both task conditions in Experiment 2. Again, the typical attentional blink effect was obtained. 
The results of this experiment show that a +1 item that contains no pattern information can cause the attentional blink. Because this +1 item contains no pattern information, it should not occupy any resources in VSTM, and as such should not cause the attentional blink. Thus the interference model cannot explain these results. On the other hand, the two-stage model can explain these results. According to this model, the white screen flash acts as a luminance mask on the target. This, then, increased the amount of time needed for second-stage processing of the target, which in turn resulted in the attentional blink.

Although both the solid white rectangle of Experiment 1 and the white screen flash of Experiment 2 produced the attentional blink effect, the effect seemed to have different durations in the two experiments. An analysis of simple main effects in Experiment 2 revealed that subjects performed better in the experimental condition than in the control condition at Position $4[F(1,16)=$ $6.505, p<.05]$, but this difference was no longer significantly different at Position $5[F(1,16)=.231]$. A similar analysis of Experiment 1 revealed that subject's performance was significantly different in the control and experimental conditions at Position $5[F(1,24)=26.796$, $p<.001]$, but this difference was no longer significantly different by Position $6[F(1,24)=2.470]$. Thus, using the white rectangle as the +1 item leads to a longer attentional blink than does using the white screen flash. Both of these items had the same luminances, so the amount of luminance masking should have been the same in the two experiments. If anything, the white screen flash should have been more effective as a luminance mask because of its larger area. Thus, on the face of it, it would seem that the two-stage model would not be able to explain this pattern of results.

This difference in timing can be explained through consideration of contour interactions between the target and the +1 stimuli in Experiments 1 and 2. As the distance between a target's contours and the contours of a luminance mask increases, the amount of masking decreases (Battersby \& Wagman, 1962). In the case of Experiment 1, the contours of the +1 item (the white rectangle) were fairly close to the target (within $1^{\circ}$ of visual angle), whereas in Experiment 2, the contours of the +1 item (the white screen flash) were widely separated from the target (separated by at least $13.4^{\circ}$ of visual angle). Thus, more masking due to contour interactions would be expected in Experiment 1 than in Experiment 2; this may account for the differences in results between Experiments 1 and 2.

\section{EXPERIMENT 3}

Metacontrast masking is a type of masking that relies on such mask-target contour interactions. In order to measure the effects of mask-target contour interactions and the effects of other types of masking, in Experiment 3 we utilized a +1 item that could act as a metacontrast mask. For this experiment, the +1 item was a hollow white rectangle. The hollow white rectangle could act as a metacontrast mask on the target because it would not overlap spatially with the target even if they were both presented at the same time (a situation that did not occur in this experiment).

\section{Method}

Subjects. Subjects were 17 undergraduate students at Johns Hopkins University who participated in order to partially fulfill a course requirement. None of the subjects in Experiment 3 had participated in the previous experiments. The apparatus and stimuli were identical to those of Experiment 1 with the following exceptions. In Experiment 3, a hollow white rectangle was presented in the +1 position, rather than a solid white rectangle. The filled-in portion of this rectangle was white (luminance $=115.9 \mathrm{~cd} / \mathrm{m}^{2}$ ), and the interior was colored in with the background color (luminance $=69.8 \mathrm{~cd} / \mathrm{m}^{2}$ ). The exterior border of the hollow rectangle subtended $3.1^{\circ}$ of visual angle both vertically and horizontally, and the interior subtended $2.5^{\circ}$ of visual angle both horizontally and vertically. The extent of the interior was such that a letter could fit inside the rectangle without touching any of the edges. The target was still green and the remaining nontarget items were black.

\section{Results and Discussion}

Figure 3 is a plot of correct probe detection as a function of probe position for both the control and experimental conditions. A repeated measures ANOVA (task condition $\times$ probe position) revealed a significant main effect of task condition $[F(1,16)=36.735, p<.001]$, a significant main effect of probe position $[F(6,96)=6.668$, $p<.001]$, and a significant task condition $\times$ probe position interaction $[F(6,96)=9.782, p<.001]$. Subjects correctly detected the probe $90.3 \%$ of the time in the control condition and $77.5 \%$ of the time in the experimental condition. Accuracy for identifying the first target was $91 \%$. Figure 3 demonstrates that, as in the previous experiments, probe detection performance was better in the con-

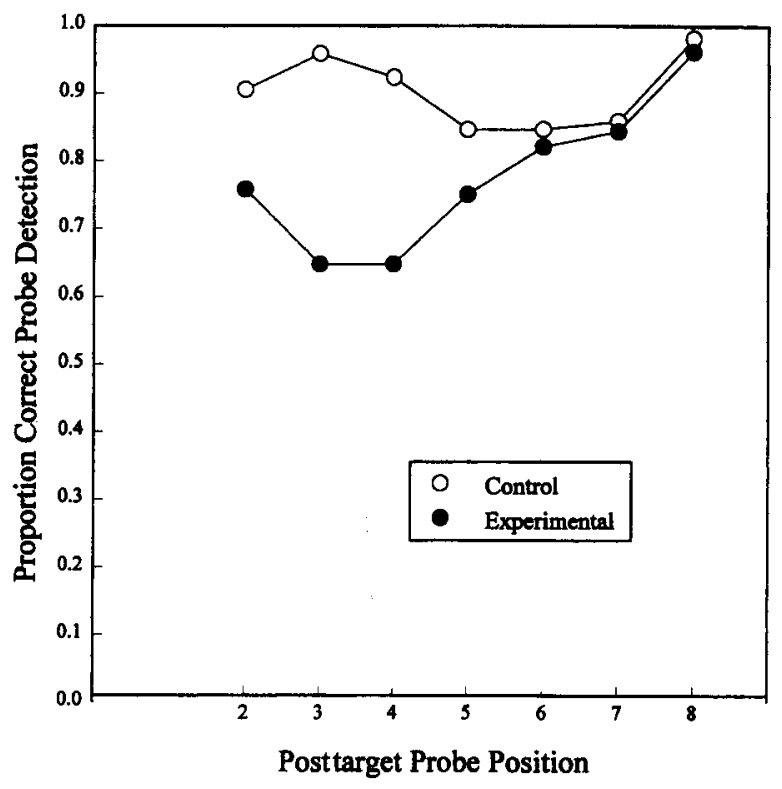

Figure 3. Mean proportion of correct probe detection trials as a function of probe position for both the experimental and the control conditions in Experiment 3. The attentional blink was also obtained in this experiment. 
trol condition than in the experimental condition. Probe position affected probe detection performance more in the experimental condition than in the control condition. This experiment thus extends previous results (which showed that luminance masking can cause the attentional blink) by demonstrating that metacontrast masking can also cause the attentional blink.

\section{EXPERIMENT 4}

So far, each type of +1 item has resulted in the attentional blink. We attribute this to the masking properties of each of these items. Another possibility is that with the timing and target configuration particular to this experiment, a +1 item is not necessary to cause the attentional blink. In order to evaluate this possibility in Experiment 4, a blank screen was used as the +1 item.

\section{Method}

Subjects. Subjects were 18 undergraduate students at Johns Hopkins University who participated in order to partially fulfill a course requirement. None of the subjects in Experiment $4 \mathrm{had}$ taken part in the previous experiments. The apparatus and stimuli were identical to those of Experiment 2 with the following exceptions. In Experiment 4, a blank screen was presented at the +1 location, whereas in Experiment 2 a white screen flash was used. The blank screen had the same luminance as the background.

\section{Results and Discussion}

Figure 4 is a plot of correct probe detection as a function of probe position for both the experimental and the control conditions. A repeated measures ANOVA (task condition $\times$ probe position) revealed a significant main

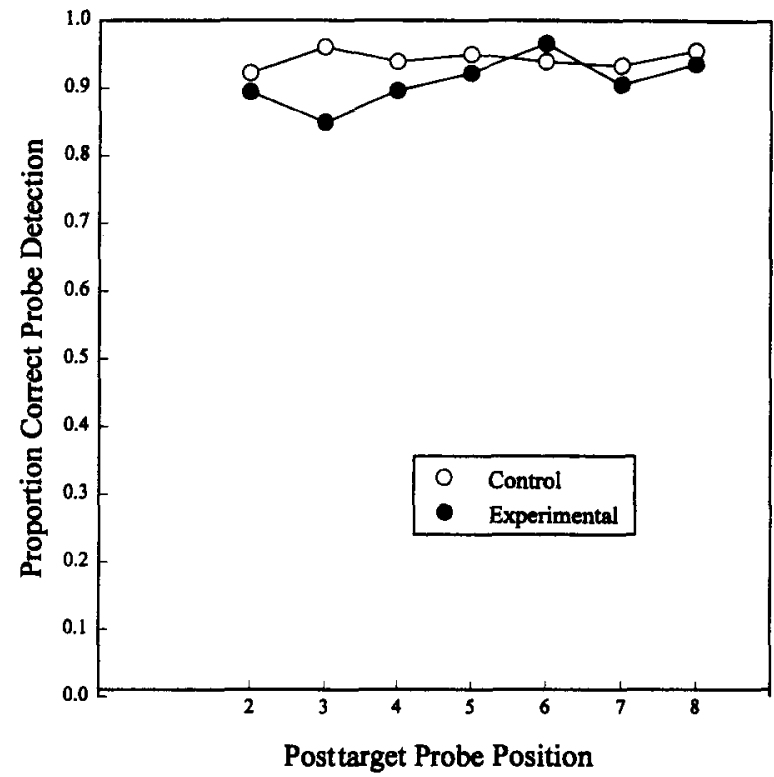

Figure 4. Mean proportion of correct probe detection trials as a function of probe position for both the experimental and the control conditions for Experiment 4. The attentional blink effect was not present. effect of task condition $[F(17,1)=5.201, p<.05]$, whereas the effect of probe position was nonsignificant $[F(6,102)=1.752]$. The interaction between task condition and probe position was also nonsignificant $[F(6,102)=1.933]$. Subjects correctly detected the probe $94.3 \%$ of the time in the control condition and $91 \%$ of the time in the experimental condition. Accuracy for identifying the first target was $96 \%$.

The lack of a significant effect of probe position and the lack of an interaction between probe position and task condition indicates that the attentional blink does not occur when the +1 item is a blank screen. There was, however, a small but significant effect of task condition. Recall that in the control condition the subject only had to perform the probe detection task, whereas in the experimental condition the subject had to first complete a target identification task before performing the probe detection task. The difference in probe detection between these two conditions might reflect the fact that it is more difficult to perform two tasks than to perform just one in isolation. This reduction in probe detection accuracy is not a result of the attentional blink because there is no significant effect of probe position.

Both the two-stage model and the interference model lead to the prediction that using a blank screen as a +1 item would eliminate the attentional blink, as noted in the introduction. The main result of Experiment 4 was the finding that using the stimulus parameters of the previous experiments, a blank screen presented as the +1 item did indeed fail to cause an attentional blink.

Previous researchers have also found that using a blank screen as a +1 item eliminates the attentional blink (Raymond et al., 1992). However, in Raymond et al.'s study, there was no main effect of task condition like the one found here. One possible reason for this discrepancy is that in the earlier study, the difference in probe detection accuracy between the experimental and the control conditions was evaluated for only two probe positions $(+3$ and +4$)$. These comparisons at Probe Position +3 and Probe Position +4 were analyzed separately. If instead the difference in probe detection performance between the control and experimental conditions had simultaneously been evaluated across all probe positions (as was done in this study), a significant effect might have been found.

\section{EXPERIMENT 5}

On the basis of the results from the second and fourth experiments, we know that using a +1 item without any pattern information will cause the attentional blink, whereas using a blank screen will not. One difference between these two conditions is that the white screen flash was a salient visual event, whereas the blank screen was not. Perhaps any salient visual event used as the +1 item will cause the attentional blink. In Experiment 5, we used $a+1$ item that was a salient visual event, but because it had a lower luminance, we hypothesized that it would not 
be an effective mask. In this experiment, the +1 item was a green screen flash.

\begin{abstract}
Method
Subjects. Subjects were 21 undergraduate students at Johns Hopkins University who participated in order to partially fulfill a course requirement. None of the subjects in Experiment 5 had participated in any of the previous experiments. The apparatus and stimuli were identical to those used in Experiment 2 with the following exceptions. In Experiment 5, a green screen flash was presented in the +1 location, rather than a white screen flash. The green screen flash and the white screen flash had the same spatial extent, but they differed in both color and luminance. The green screen flash had a luminance of $57.2 \mathrm{~cd} / \mathrm{m}^{2}$, and the white screen flash had a luminance of $115.9 \mathrm{~cd} / \mathrm{m}^{2}$. Also, in Experiment 2, a green target was followed by a white screen flash. In order to preserve the amount of similarity between the target and the +1 item, a white target was used with the green screen flash in this experiment; the other items in the stream remained black.
\end{abstract}

\section{Results and Discussion}

Figure 5 shows probe detection accuracy plotted as a function of probe position. A repeated measures ANOVA revealed a significant main effect of task condition $[F(1,20)=9.813, p<.01]$. The effect of probe position was nonsignificant $[F(6,120)=1.163]$, and the interaction between task condition and probe position was also nonsignificant $[F(6,120)=2.165]$. Subjects correctly detected the probe $90.3 \%$ of the time in the control condition and $84.1 \%$ of the time in the experimental condition. Accuracy for identifying the first target was $97 \%$.

The lack of a significant effect of probe position and the lack of an interaction between probe position and task condition indicates that the attentional blink does not occur when the +1 item is a green screen flash. The inter-

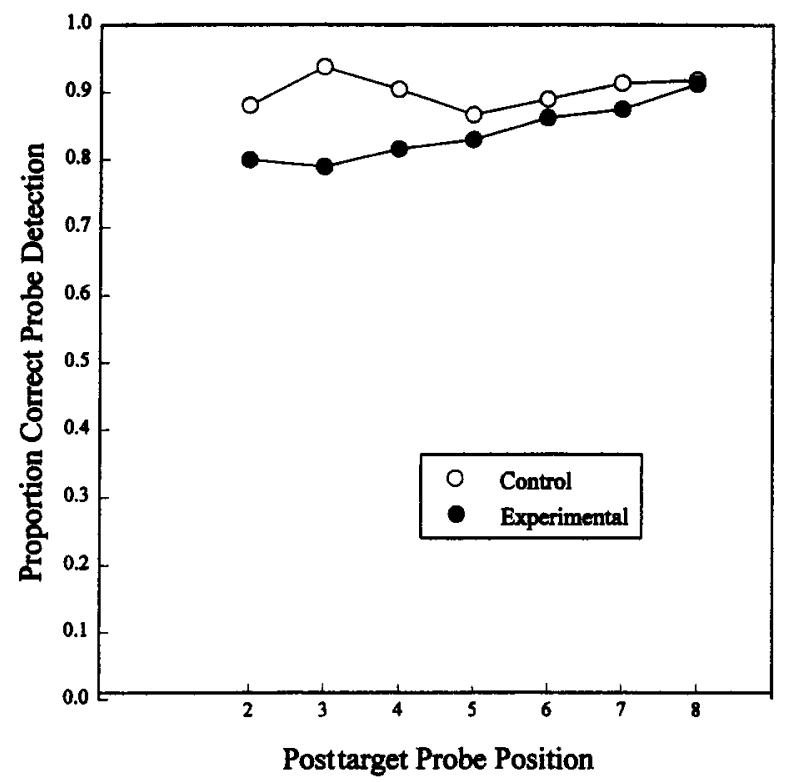

Figure 5. Mean proportion of correct probe detection trials as a function of probe position for both the experimental and control conditions for Experiment 5. The attentional blink effect was absent for Experiment 5. ference model would predict that the green screen would not cause the attentional blink because it is not visually similar to the probe. However, the interference model cannot explain why a white screen flash following a green target would cause the attentional blink, whereas a green screen flash following a white target would not. According to the two-stage model, the white screen flash would cause the blink because it is an effective mask. Because the green screen flash has a lower luminance, it would not be as effective a mask and therefore would not cause the attentional blink. The main finding of Experiment 5 was that not every salient visual event used in the +1 position will cause an attentional blink.

\section{GENERAL DISCUSSION}

In the five experiments reported in this paper, subjects viewed an RSVP stream of letters and the nature of the +1 item was varied. Previous results and the results from the pilot study demonstrate that when the +1 item is a pattern mask, the attentional blink occurs. Experiments 1 and 2 showed that the attentional blink also occurs when the +1 item is a luminance mask such as a white rectangle or a white screen flash. Experiment 3 showed that a metacontrast mask in the +1 position also causes the attentional blink. Experiment 4 showed that the attentional blink does not occur in the absence of a +1 item, and Experiment 5 showed that not every +1 item that represents a salient visual event will cause the attentional blink.

The magnitude of the attentional blink for a given experiment was determined by calculating the difference between $100 \%$ and the percentage of correct probe detection in the experimental condition at each serial position. The values for Positions +2 through +8 were summed, and then the totals were averaged across subjects. An ANOVA performed on the magnitudes of the blink across all experiments revealed a significant main effect of experiment $[F(4,93)=5.651, p<.001]$. The blink magnitude in the white rectangle, white screen flash, and metacontrast experiments was significantly larger than that in the blank screen and green screen flash experiments $[F(1,96)=17.105, p<.001]$.

According to the interference model, the +1 item causes the attentional blink by occupying limited resources in VSTM. This limits the weighting the probe can receive, causing errors in its retrieval. As items become more similar to the probe, they occupy more resources. A +1 item that is visually similar to the probe, such as a dot pattern (Raymond et al., 1995), would therefore be expected to cause the attentional blink. The interference model would not necessarily predict that using a white rectangle as the +1 item would lead to the attentional blink, but it might explain this result after the fact by assuming that the white rectangle contains some pattern information and therefore occupies VSTM resources. Raymond et al. (1995) assumed that when the +1 item is a blank screen, no attentional blink is produced because the blank screen contains no pattern information. The interference model cannot, however, explain how a +1 item 
that has no pattern information can cause the attentional blink, as was the case in Experiment 2 (white screen flash). It is possible to explain this result in the same basic framework if one assumes that any visual feature that differs from the background (in this case, increased luminance) can occupy resources in VSTM. Even this revised account would still not be able to explain why a white screen flash in the +1 position would cause the attentional blink, whereas a green screen flash in the same position would not. Therefore, this pattern of results is problematic for the interference model.

These results can be accounted for rather nicely by the Chun and Potter (1995) two-stage model. In this model, short-lived representations of the experimental stimuli are formed in the first stage, and these representations undergo further processing in a limited-capacity second stage. If the +1 item can act as an effective mask of the target, it will prolong second-stage processing of the target. This will in turn delay second-stage processing of the probe. If the first-stage representation of the probe has faded before the second stage processor is available, the probe will not be identified. This two-stage model explains the experimental results in the following way. In Experiments 1 and 2, the white rectangle and the white screen flash acted as luminance masks that increased the time needed to complete second-stage processing of the target. The results from Experiment 3 can be explained in the same way except that the hollow white rectangle acted as a metacontrast mask. Clearly, the blank screen cannot act as an effective mask and so no blink would be expected. The question remains why a green screen flash would not produce the blink, whereas a white screen flash would. The answer lies in the fact that the green screen flash had a lower luminance than the white screen flash did, and thus should be less effective as a mask. If the green screen flash is less effective as a mask than the white screen flash, target identification should be better, which it is [ $97 \%$ accuracy with the green screen flash vs. $94 \%$ accuracy with the white screen flash, $F(1,36)=5.720, p<$ $.05]$.

According to Shapiro et al. (1994), one of the predictions of a masking model of the attentional blink is that the difficulty of processing the first target should be positively correlated with the magnitude of the attentional blink. ${ }^{1}$ Shapiro et al. made one such comparison between the $d^{\prime}$ 'value of the target's detectability and blink magnitude. For each experiment, two values were calculated, a mean $d^{\prime}$ and a mean blink magnitude. They first analyzed this correlation across the experiments that produced the attentional blink effect and found a nonsignificant correlation. Shapiro et al. took this as evidence that the magnitude of the blink is not related to the difficulty of the target task. The lack of statistical significance is not really surprising considering the small number of experiments that entered into this correlation (only four experiments were used), resulting in a comparison with two degrees of freedom. In fact, when Shapiro et al. increased their power by including both those experiments that produced the blink and those that did not, they found a significant correlation.

In order to elucidate the relationship between target identification accuracy and blink magnitude, we performed a similar analysis on our data. For our data, the prediction is that target identification accuracy should be negatively correlated with blink magnitude. Blink magnitude was calculated the same way as in Raymond et al. (1995). The correlation between the mean target accuracy and the mean blink magnitude for all five experiments revealed a nonsignificant and negative correlation $[r(3)=$ $-.8, p>.1]$. Even though this relationship was not significant, low statistical power might have rendered the test unable to detect a real effect.

Two factors play a role in determining the target identification accuracy for a given subject. One factor is the experimental condition in which the subject served. For example, the average target identification accuracy for Experiment 5 was $97 \%$, whereas the average target identification accuracy for Experiment 1 was $88 \%$. In addition, the perceptual abilities of the individual subjects vary, and therefore target identification accuracy will vary within a given experiment. If it is true that target identification accuracy is negatively correlated with blink magnitude, this should hold true not only between experiments but also within experiments. In order to test this and to increase our statistical power, we determined the overall correlation between target identification accuracy and blink magnitude across all of the subjects in the five experiments. This produced a significant negative correlation between target identification accuracy and blink magnitude $[r(96)=-.48, p<.01]$. This correlation was increased slightly when we used only the data from the experiments in which the blink was produced $[r(57)=$ $-.49, p<.01]$. These results lead us to conclude that target identification accuracy and blink magnitude are negatively correlated and that Shapiro et al. (1994) did not find this effect because their comparison lacked sufficient statistical power. A more extensive comparison between target identification accuracy and blink magnitude was made by Seiffert and DiLollo (1995), who took the target identification accuracies and the blink magnitudes from five separate publications (a total of 27 experiments). They found a significant negative correlation between the two values, suggesting that the inverse relationship between target identification accuracy and blink magnitude found in this study is a general property of the attentional blink.

In conclusion, our results show that +1 items that act as effective masks produce the attentional blink, whereas those that are not effective masks do not produce the attentional blink. The presentation of patterned stimuli in close temporal proximity to the target is not a necessary condition for the attentional blink. A +1 item that contains no pattern information can still cause the attentional blink if it has masking properties. These results are consistent with the Chun and Potter (1995) two-stage model of the attentional blink, in which the +1 item masks the 
target. Note that these results do not preclude the possibility that visual similarity between the +1 item and the target or probe can influence the attentional blink. Rather, they indicate that such similarity is not necessary in order for the attentional blink to occur.

\section{REFERENCES}

Battersby, W. S., \& Wagman, I. H. (1962). Neural limitations of visual excitability: IV. American Journal of Physiology, 203, 359-365.

BREITMEYER, B. (1984). Visual masking: An integrative approach. New York: Oxford University Press.

Broadbent, D. E., \& Broadbent, M. H. P. (1987). From detection to identification: Response to multiple targets in rapid serial visual presentation. Perception \& Psychophysics, 42, 105-113.

Chun, M. M., \& Potter, M. C. (1995). A two-stage model for multiple target detection in rapid serial visual presentation. Journal of Experimental Psychology: Human Perception \& Performance, 21, 109-127.

Duncan, J., \& Humphreys, G. W. (1989). Visual search and stimulus similarity. Psychological Review, 96, 433-458.

Ghirardelli, T. G., Grandison, T. D., \& Egeth, H. E. (1995). Posttarget masking and the attentional blink. Investigative Ophthalmology \& Visual Science, 36(Suppl.), 1751.

Raymond, J. E., Shapiro, K. L., \& Arnell, K. M. (1992). Temporary suppression of visual processing in an RSVP task: An attentional blink? Journal of Experimental Psychology: Human Perception \& Performance, 18, 849-860.

Raymond, J. E., Shapiro, K. L., \& Arnell, K. M. (1995). Similarity determines the attentional blink. Journal of Experimental Psychology: Human Perception \& Performance, 21, 653-662.

Seiffert, A. E., \& Dilollo, V. (in press). Low-level masking in the attentional blink. Journal of Experimental Psychology: Human Perception \& Performance.
Shapiro, K. L., RaYmond, J. E., \& ARnell, K. M. (1994). Attention to visual pattern information produces the attentional blink in RSVP. Journal of Experimental Psychology: Human Perception \& Performance, 20, 357-371.

STERnBERG, S. (1967). Two operations in character recognition: Some evidence from reaction-time measurements. Perception \& Psychophysics, 2, 45-53.

Weichselgartner, E., \& Sperling, G. (1987). Dynamics of controlled visual attention. Science, 238, 778-780.

WhITE, C. W., \& LORBER, C. M. (1976). Spatial-frequency specificity in visual pasking. Perception \& Psychophysics, 19, 281-284.

\section{NOTE}

1. This argument might not necessarily be true for cases in which target identification accuracy is close to ceiling. The presence of a mask can affect performance of a character identification task in two ways. The mask might reduce accuracy and/or increase the amount of time needed to process the character. In regard to models of the attentional blink, the effects a mask has on processing time may be more important than the effects the mask has on identification accuracy. For example, in a speeded character identification task, the presence of a mask affected reaction times (this is assumed to be an approximation of processing time) without substantially altering the low error rate (Sternberg, 1967). It seems possible that in some instances a mask could have a large effect on the magnitude of the attentional blink by changing the amount of processing time necessary without substantially altering target identification accuracy. Thus the lack of an inverse relationship between target identification accuracy and blink magnitude would not necessarily rule out effects of masking.

(Manuscript received November 10, 1995; revision accepted for publication April 14, 1996.) 